\title{
SOLUÇÃO ANALÍTICA E VALIDAÇÃO EXPERIMENTAL DE UM MODELO PARA HIDRATAÇÃO DE SOJA COM COEFICIENTE DE TRANSFERENCIA DE MASSA VARIÁVEL
}

\author{
D. J. NICOLIN ${ }^{1}$, R. MENECHINI NETO ${ }^{1}$, R. M. M. JORGE ${ }^{2}$, L. M. M. JORGE ${ }^{1}$ \\ ${ }^{1}$ Universidade Estadual de Maringá, Departamento de Engenharia Química, Programa de Pós- \\ graduação em Engenharia Química \\ ${ }^{2}$ Universidade Federal do Paraná, Departamento de Engenharia Química \\ E-mail para contato: douglas.nicolin@gmail.com/lmmj@deq.uem.br
}

\begin{abstract}
RESUMO - A hidratação de grãos de soja foi modelada matematicamente por meio de um modelo de parâmetros concentrados obtido por um balanço de massa. No balanço o acúmulo de água nos grãos é causado por convecção natural. Para refletir a queda do coeficiente de transferência de massa conforme a concentração de água nos grãos aumenta, o que causa a diminuição da transferência de massa, propôs-se que o coeficiente varia linearmente com umidade. Até então este modelo só havia sido resolvido numericamente, desconhecendo-se a solução analítica. A solução analítica do modelo foi obtida, comparada com a solução numérica e validada frente a dados experimentais de umidade em função do tempo para soja do cultivar CD 202 em várias temperaturas, confirmando que a solução analítica é correta e pode ser utilizada na simulação, análise, otimização e projeto de unidades de hidratação. Os parâmetros ajustados com a solução numérica e analítica foram idênticos e aumentaram exponencialmente com a temperatura.
\end{abstract}

\section{INTRODUÇÃO}

Uma das grandes motivações para processamento de grãos de soja está em fortalecer a produção e o desenvolvimento de novos tipos de produtos alimentícios, nutricionais, farmacêuticos, cosméticos e industriais, incluindo co-produtos e ingredientes para aplicações específicas que utilizem algum componente proveniente da soja (GURIQBAL, 2010). Em termos de processamento de soja com o objetivo de produzir alimentos, a hidratação constitui uma parte importante do processo de extração de proteínas sendo um pré-tratamento dos grãos que tem como objetivo alterar as características texturais dos grãos e facilitar a extração das proteínas(CIABOTTI et al., 2009; COUTINHO et al., 2010; NICOLIN, 2012; NICOLIN et al., 2013; PAN e TANGRATANAVALEE, 2003). Portanto, a compreensão da cinética de hidratação é um fator muito importante para estabelecer uma relação precisa entre a umidade que os grãos absorvem e o tempo de hidratação.

Os modelos empíricos são uma alternativa na modelagem matemática da hidratação de grãos. Os modelos de Peleg(1988), Singh e Kulshrestha(1987) e Pilosof, Boquet e Bartholomai(1985) são importantes modelos empíricos vastamente utilizados para a descrição da hidratação de produtos alimentícios. Embora os modelos empíricos forneçam bons ajustes de modo geral, eles possuem a desvantagem de não consideram etapas elementares de transferência e são representações diretas de dados experimentais (BEQUETTE, 1998; PINTO e LAGE, 2001). 
Modelos fenomenológicos são aqueles que consideram pressupostos teóricos e etapas elementares de transferência de massa, logo são mais vantajosos que os modelos empíricos quando o entendimento mais detalhado do processo estudado está em foco (BEQUETTE, 1998). Alguns autores propuseram modelos fenomenológicos de parâmetros concentrados, nos quais não são consideradas variações espaciais das variáveis de interesse, para a descrição da hidratação de soja(COUTINHO et al., 2005, 2007) e de ervilha (OMOTO et al., 2009). Para a hidratação de soja os autores propuseram em seus dois trabalhos um modelo que partiu do mesmo balanço resultante de um balanço de massa global no grão em que o acúmulo de água é causado pela convecção ao redor do grão e em ambos foram consideradas maneiras diferentes de se incluir a variação tanto do coeficiente de transferência de massa quanto do volume dos grãos durante da hidratação. Nestes trabalhos o coeficiente de transferência de massa foi considerado como função da umidade para refletir a diminuição que esta propriedade sofre conforme a umidade se aproxima do equilíbrio.Para modelar a hidratação de ervilha os autores propuseram um modelo de parâmetros concentrados que partiu do mesmo balanço de massa dos trabalhos de Coutinho et al. $(2005,2007)$. O fluxo convectivo foi considerado com coeficiente de transferência de massa constante. Neste trabalho o autor considera o volume dos grãos de ervilha constante e obtém uma solução analítica para o modelo proposto.

Neste contexto o objetivo deste trabalho foi propor um modelo fenomenológico de parâmetros concentrados que possuísse o coeficiente de transferência de massa dependente da concentração de água nos grãos, para considerar a diminuição do valor deste parâmetro conforme a hidratação se aproxima do equilíbrio. O modelo foi resolvido analiticamente e a solução analítica obtida foi comparada com a solução numérica do modelo e validada com dados experimentais de hidratação de soja do cultivar CD 202. Os parâmetros do modelo foram ajustados pelo método dos mínimos quadrados e apresentaram os mesmos valores tanto para a solução numérica quanto para a solução analítica. Os parâmetros apresentaram aumento exponencial em função da temperatura.

\section{TEORIA}

A Equação 1 apresenta o modelo obtido por um balanço de massa global no grão de soja. O sistema foi considerado como de parâmetros concentrados e o acúmulo de água no sistema foi considerado como sendo causado predominantemente por convecção. O fluxo convectivo é dado por $N_{A}=K_{S}\left(\rho_{e q}-\rho_{A}\right)$. Nesta equação o grão é considerado esférico e seu volume é considerado constante ao longo de todo o processo de hidratação.

$$
V \frac{d \rho_{A}}{d t}=A K_{S}\left(\rho_{e q}-\rho_{A}\right)
$$

Substituindo as equações para o volume $\left(V=(4 / 3) \pi R^{3}\right)$, para a área superficial $\left(A=4 \pi R^{2}\right)$ da esfera e para a dependência linear do coeficiente de transferência de massa com a concentração de água nos grãos $\left(K_{S}=a+b \rho_{A}\right)$ é possível obter a forma final do modelo que é apresentada pela Equação 2. Além da equação diferencial do modelo, esta equação também apresenta a condição inicial necessária para a solução sua solução. A condição inicial estabelece que no início da hidratação $(t=0)$ a umidade nos grãos é uniformemente distribuída em todos os grãos e conhecida. 


$$
\begin{aligned}
& \frac{d \rho_{A}}{d t}=\frac{3}{R}\left(a+b \rho_{A}\right)\left(\rho_{e q}-\rho_{A}\right) \\
& \rho_{A}(0)=\rho_{A 0}
\end{aligned}
$$

A Equação 2 é uma equação diferencial de variáveis separáveis e, para sua integração, pode ser escrita na seguinte forma:

$$
\frac{d \rho_{A}}{\left(a+b \rho_{A}\right)\left(\rho_{e q}-\rho_{A}\right)}=\frac{3}{R} d t
$$

A fração presente no termo do lado esquerdo da Equação 3 não pode ser integrado diretamente como é possível fazer com o termo do lado direito da mesma equação. Para simplificar a fração do lado esquerdo da Equação 3 utilizou-se a sua expansão por frações parciais como é apresentado na Equação 4.

$$
\frac{1}{\left(a+b \rho_{A}\right)\left(\rho_{e q}-\rho_{A}\right)}=\frac{A^{\prime}}{\left(a+b \rho_{A}\right)}+\frac{B^{\prime}}{\left(\rho_{e q}-\rho_{A}\right)}
$$

Quando se resolve esta expansão por frações parciais é possível obter as constantes $A^{\prime}$ e $B^{\prime}$ como sendo $A^{\prime}=b /\left(a+b \rho_{e q}\right)$ e $B^{\prime}=1 /\left(a+b \rho_{e q}\right)$. Uma vez que se conhecem as constantes das frações parciais é possível substitui-las na Equação 3 para fornecer uma equação que pode ser integrada imediatamente.

$$
\frac{b}{\left(a+b \rho_{e q}\right)} \int \frac{d \rho_{A}}{\left(a+b \rho_{A}\right)}+\frac{1}{\left(a+b \rho_{e q}\right)} \int \frac{d \rho_{A}}{\left(\rho_{e q}-\rho_{A}\right)}=\frac{3}{R} \int d t
$$

O resultado da Equação 5 pode ser simplificado e é apresentado pela Equação 6 em função da constante de integração que aparece após o procedimento de integração.

$$
\frac{1}{\left(a+b \rho_{e q}\right)} \ln \left(\frac{a+b \rho_{A}}{\rho_{e q}-\rho_{A}}\right)=\frac{3}{R} t+C_{1}
$$

Utilizando-se a condição inicial presente na Equação 2 é possível encontrar a constante de integração $C_{1}$, que pode ser substituído na Equação 6 e o resultado, de maneira devidamente rearranjada, fornece a solução analítica para o modelo proposto (Equação 7).

$$
\begin{aligned}
& \rho_{A}(t)=\frac{-a+\rho_{e q} K_{1} e^{K_{2} t}}{b+K_{1} e^{K_{2} t}} \\
& \text { sendo } K_{1}=\frac{a+b \rho_{A 0}}{\rho_{e q}-\rho_{A 0}} \text { e } K_{2}=\frac{3\left(a+b \rho_{e q}\right)}{R} \text {. As constantes } a \text { e } b \text { são os parâmetros que }
\end{aligned}
$$

caracterizam a dependência linear de $K_{S} \operatorname{com} \rho_{A}$ e são os parâmetros que foram ajustados.

\section{MATERIAIS E MÉTODOS}

Para a obtenção dos dados experimentais de umidade em função do tempo foram utilizados grãos de soja do cultivar CD 202 fornecidos pela Cooperativa Agropecuária MourãoenseLtda (COAMO). Inicialmente pesou-se aproximadamente $300 \mathrm{~g}$ de soja. 
Preparou-se o banho termostático (Marconi MA184) para o controle da temperatura de hidratação. As temperaturas de hidratação utilizadas foram 10, 20, 30, 40 e $50^{\circ} \mathrm{C}$. Enquatno o banho termostática atingia a temperatura desejada, 1,5 litros de uma solução de benzoato de sódio foi preparada para a imersão dos grãos. Benzoato de sódio foi usado na água de hidratação como agente conservante, ou seja, para evitar a proliferação de microorganismos. A solução de benzoato de sódio foi inserida num recipiente que foi inserido no banho termostático para que a solução também atingisse a temperatura desejada. Uma vez que a temperatura de hidratação de interesse foi atingida, os grãos de soja foram inseridos no banho e iniciou-se a contagem do tempo. Em tempos pré-determinados foram retiradas amostras de soja que tiveram o excesso de umidade superficial retirado com papel toalha. As amostras foram pesadas e inseridas em estufa (Fanem $315 \mathrm{SE}$ ) durante $24 \mathrm{~h}$ a $105^{\circ} \mathrm{C}$ para obtenção da umidade de cada amostra (LUTZ, 1985).

Foi desenvolvida uma rotina em MATLAB para a solução numérica do modelo com o intuito de verificar a validade da solução analítica obtida na seção 2. Para a integração numérica do modelo foi utilizada a rotina "ode45" do MATLAB que é baseada da fórmula de Runge-Kutta (4,5) (DORMAND e PRINCE, 1980). A obtenção dos melhores valores dos parâmetros do modelo foi feita pelo método dos mínimos quadrados por meio da minimização de uma função-objetivo quadrática (Equação 8). O método de otimização dos parâmetros usado foi o método de Levenberg-Marquardt(LEVENBERG, 1944; MARQUARDT, 1963) que está presente no MATLAB no comando "nlinfit".

$$
\phi=\sum_{i=1}^{N}\left(\rho_{\text {Acalc }}^{i}-\rho_{\text {Aexp }}^{i}\right)^{2}
$$

\section{RESULTADOS E DISCUSSÃO}

A Tabela 1 apresenta os valores da função objetivo ao final do procedimento iterativo de ajuste, feito com o uso do comando "nlinfit". Todos os valores de $\phi$ tiveram ordem de grandeza de $10^{-2} \mathrm{~g}^{2} / \mathrm{cm}^{6}$, indicando bons ajustes uma vez que a função objetivo $\phi$ representa a o distanciamento quadrático entre os valores calculados pelo modelo e os valores experimentais. Tanto os ajustes feitos com a solução numérica quanto com a solução analítica forneceram valores idênticos de $\phi$.

Tabela 1: Resíduo quadrático para todos os ajustes

\begin{tabular}{|c|c|}
\hline $\mathrm{T}\left({ }^{\circ} \mathrm{C}\right)$ & $\phi\left(\mathrm{g}^{2} / \mathrm{cm}^{6}\right)$ \\
\hline 10 & 0,0479 \\
\hline 20 & 0,0236 \\
\hline 30 & 0,0363 \\
\hline 40 & 0,0412 \\
\hline 50 & 0,0302 \\
\hline
\end{tabular}

Os parâmetros do modelo, $a$ e $b$, foram satisfatoriamente ajustados (Figura 1) possuindo baixos valores de desvio padrão, sendo que o desvio padrão para cada valor de cada parâmetro foi indicado na Figura 1 por barras verticais. Os desvios foram menores para as duas primeiras temperaturas e maiores para as demais. Os parâmetros $a$ e $b$ aumentaram 
exponencialmente com a temperatura e uma função exponencial foi satisfatoriamente ajustada para descrever este comportamento na faixa experimental considerada. O parâmetro $b$ foi apresentado na figura como $-b$ para facilitar a visualização e a função exponencial para ele também foi apresentada com seu negativo. Vale ressaltar que os valores ajustados para os parâmetros foram idênticos tanto se utilizando a solução numérica quanto a solução analítica.

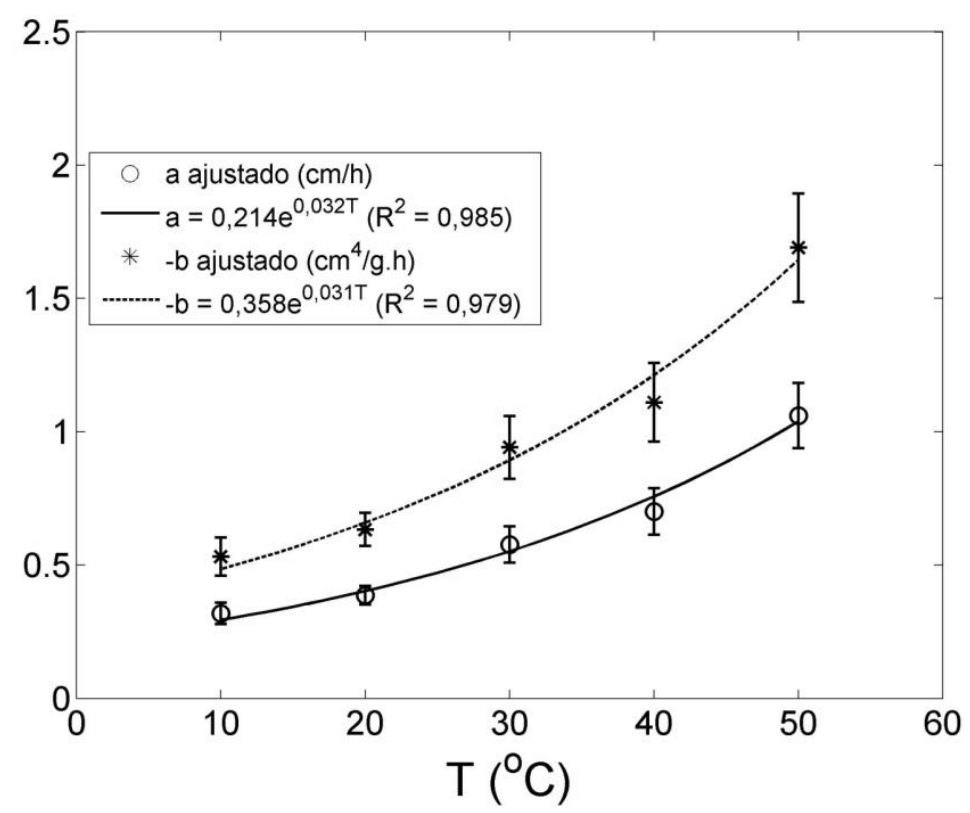

Figura 1: Comportamento dos parâmetros em função da temperatura.

A Figura 2 apresenta o modelo resolvido pela solução numérica e pela solução analítica para as temperaturas de 10,30 e $50^{\circ} \mathrm{C}$. É possível observar nesta figura que a solução analítica e a solução numérica se sobrepõem em toda a faixa de tempo apresentada. Isto indica que a solução analítica é realmente correta e válida para representar o processo de hidratação. Optou-se por apresentar os modelos para um tempo menor de hidratação para facilitar a visualização do comportamento das soluções. Para tempos maiores de hidratação a sobreposição da solução numérica e analítica se mantém.

A comparação da solução numérica e analítica com dados experimentais é apresentada na Figura 3. Novamente pode ser observado que as soluções se sobrepõem em toda a faixa de tempo considerada, indicando, mais uma vez, a validade da solução analítica obtida frente à solução numérica do modelo proposto. Ambas as soluções representam satisfatoriamente as principais tendências dos dados experimentais. Para fins de clareza na exposição dos resultados, optou-se por apresentar na Figura 3 somente as soluções numérica e analítica e os dados experimentais para a temperatura de hidratação de $10^{\circ} \mathrm{C}$. A sobreposição das soluções numérica e analítica e a representação das principais tendências dos dados experimentais se mantiveram para as demais temperaturas. 


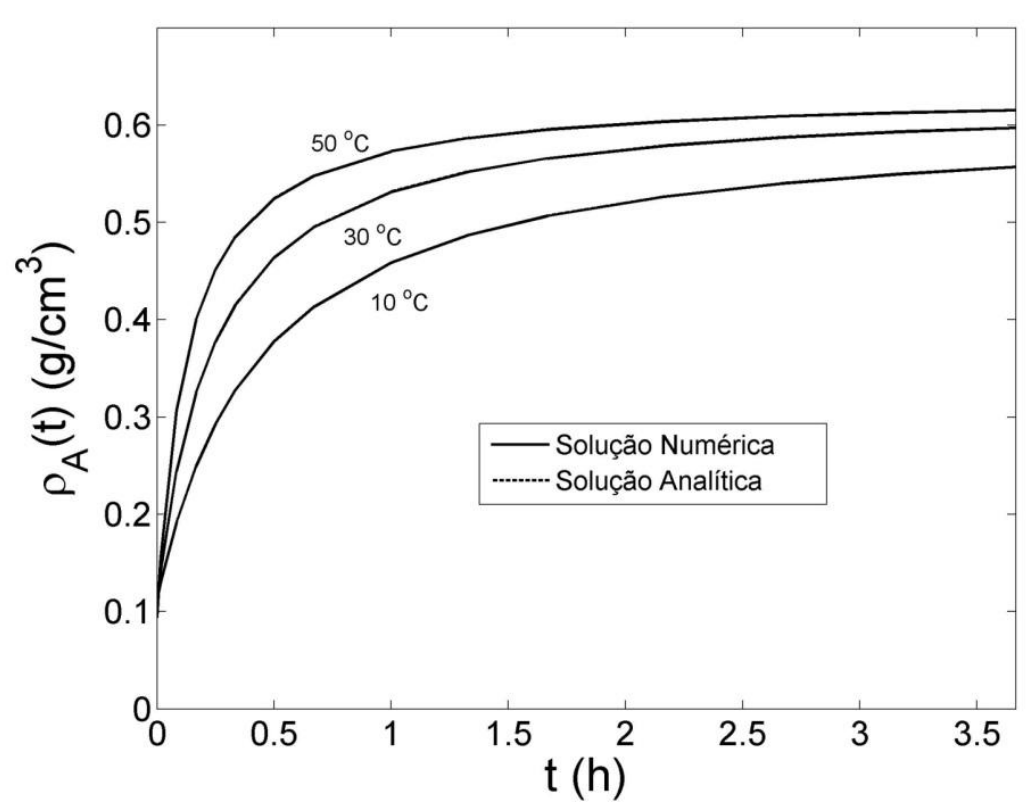

Figura 2: Solução numérica e analítica para diferentes temperaturas.

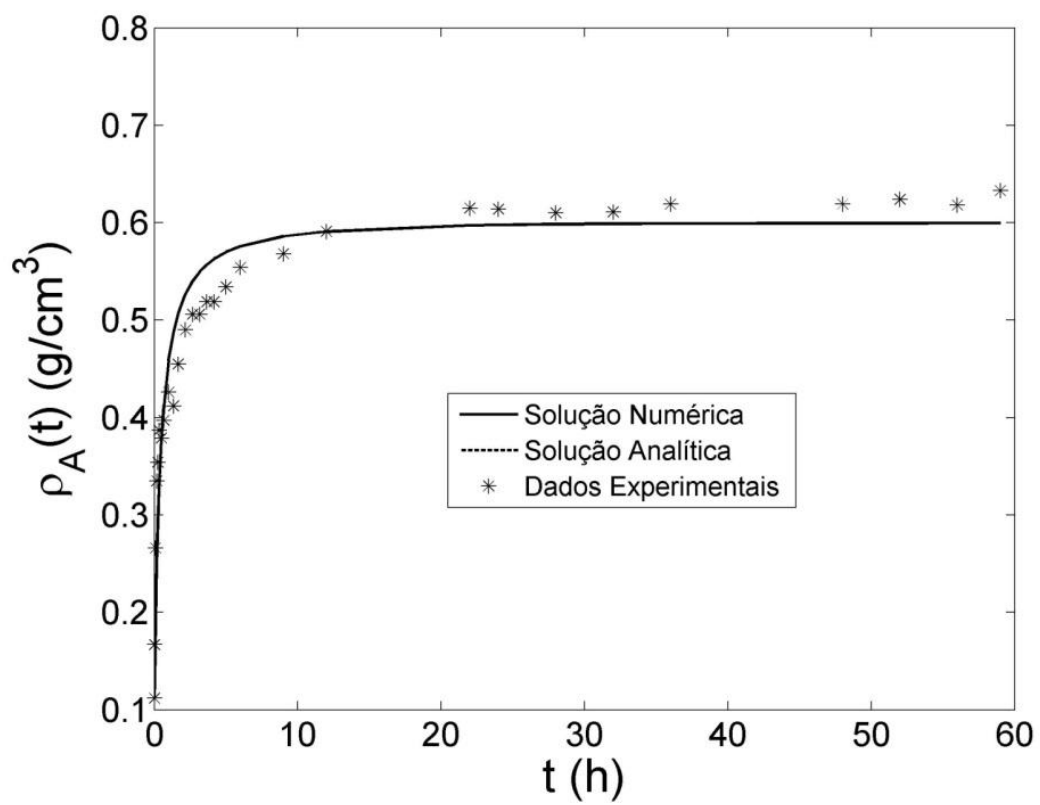

Figura 3: Solução numérica, analítica e dados experimentais para $\mathrm{T}=10^{\circ} \mathrm{C}$.

A Figura 4 apresenta o comportamento do coeficiente de transferência de massa $\left(K_{S}\right)$ em função da concentração de água nos grãos $\left(\rho_{A}\right)$ obtido a partir do ajuste dos parâmetros tanto para a solução numérica quanto para a solução analítica. Nas três temperaturas utilizadas, $K_{S}$ decaiu em função de $\rho_{A}$ para refletir o fato de que, em concentrações de água mais próximas à concentração de equilíbrio $\left(\rho_{e q}\right)$ a transferência de massa tende a cessar pela ausência do gradiente de umidade para que ela aconteça. Temperaturas mais altas fornecem valores de $K_{S}$ maiores, indicando que a transferência de massa ocorre mais rapidamente para temperaturas mais altas. 


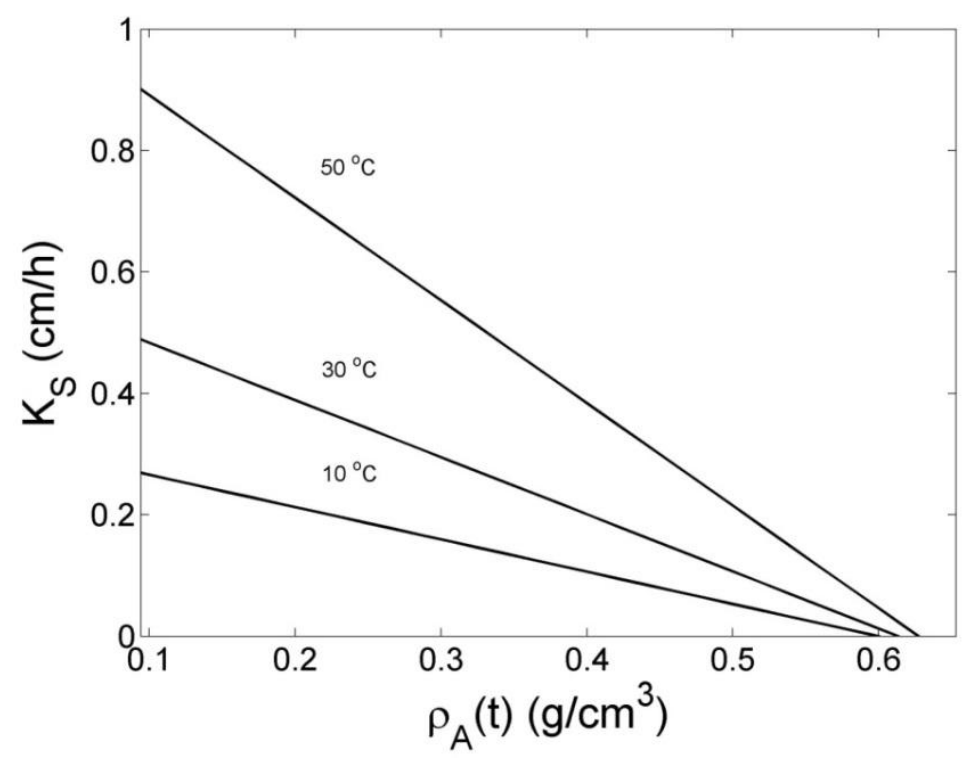

Figura 4: $K_{S}$ em função de $\rho_{A}$ para várias temperaturas.

\section{CONCLUSÕES}

Com base nos resultados apresentados é possível concluir que a solução analítica apresentada para o modelo de parâmetros concentrados proposto é válida para a descrição da hidratação de grãos de soja na faixa experimental considerada. $O$ fato da solução numérica se sobrepor à analítica,de ambas representarem as tendências dos dados experimentais satisfatoriamente e dos parâmetros ajustados a ambas as soluções serem idênticos contribuem para a validade da solução analítica obtida.

Os parâmetros foram ajustados satisfatoriamente, pois o ajuste forneceu valores baixos para a função objetivo ao fim do processo de otimização. Os parâmetros ajustados, $a$ e $b$, aumentaram exponencialmente com a temperatura e funções para ambos foram satisfatoriamente ajustadas para a descrição de tal comportamento.

A partir da obtenção dos parâmetros do modelo foi possível calcular o comportamento do coeficiente de transferência de massa $\left(K_{S}\right)$ em função da concentração de água no interior dos grãos $\left(\rho_{A}\right)$. O comportamento obtido corresponde ao esperado uma vez que $K_{S}$ deve diminuir com o aumento de $\rho_{A}$ para refletir a diminuição da transferência de massa para valores de $\rho_{A}$ próximos ao estado de equilíbrio.

\section{NOMENCLATURA}

$a \quad$ Constante de $K_{S}$ linear $(\mathrm{cm} / \mathrm{h})$

b Constante de $K_{S}$ linear $\left(\mathrm{cm}^{4} / \mathrm{g} . \mathrm{h}\right)$

$A$ Área superficial dos grãos $\left(\mathrm{m}^{2}\right)$

$A^{\prime} \quad$ Constante, frações parciais

$B^{\prime} \quad$ Constante, frações parciais

$C_{1} \quad$ Constante de integração

$i \quad$ Índice de somatório

$K_{S} \quad$ Coeficiente de transferência de massa $(\mathrm{cm} / \mathrm{h})$
$N \quad$ Número máximo de valores no somatório

$N_{A} \quad$ Fluxo de água $\left(\mathrm{g} / \mathrm{cm}^{2} . \mathrm{h}\right)$

$R \quad$ Raio dos grãos (cm)

$t \quad$ Tempo (h)

$T$ Temperatura $\left({ }^{\circ} \mathrm{C}\right)$

$V \quad$ Volume dos grãos $\left(\mathrm{m}^{3}\right)$

Símbolos gregos

$\rho_{A} \quad$ Concentração de água $\left(\mathrm{g} / \mathrm{cm}^{3}\right)$

$\rho_{\text {Acalc }}^{i}$ Concentrações calculadas pelo modelo $\left(\mathrm{g} / \mathrm{cm}^{3}\right)$

$\rho_{\text {Aexp }}^{i}$ Concentrações experimentais $\left(\mathrm{g} / \mathrm{cm}^{3}\right)$ 
$\rho_{A 0}$ Concentração de água inicial $\left(\mathrm{g} / \mathrm{cm}^{3}\right)$

$\phi \quad$ Função objetivo $\left(\mathrm{g}^{2} / \mathrm{cm}^{6}\right)$

$\rho_{e q} \quad$ Concentração de água no equilíbrio $\left(\mathrm{g} / \mathrm{cm}^{3}\right)$

\section{REFERÊNCIAS BIBLIOGRÁFICAS}

BEQUETTE, B. W. Process Dynamics Modeling, Analysis and Simulation. 1st Editio ed. New Jersey: Prentice-Hall PTR, 1998. p. 621

CIABOTTI, S.;BARCELOS, M. F. P.; CIRILlO, M. A.; PINHEIRO, A. C. M. Propriedades tecnológicas e sensoriais de produto similar ao tofu obtido pela adição de soro de leite ao extrato de soja Sensorial and technologic properties of product similar to tofu obtained with whey and soymilk addiction. Ciencia e Tecnologia de Alimentos, v. 29, n. 2, p. 346-353, 2009.

COUTINHO, M. R.;OMOTO, E. S.; ANDRADE, C. M. G.; JORGE, L. M. M.. Modelagem e validação da hidratação de grãos de soja. Ciência e Tecnologia de Alimentos, v. 25, n. 3, p. 603610, doi:10.1590/S0101-20612005000300034, 2005.

COUTINHO, M. R.; CONCEIÇÃO, W. A. S.; OMOTO, E. S.; ANDRADE, C. M. G.; JORGE, L. M. M. Novo modelo de parâmetros concentrados aplicado à hidratação de grãos. Ciência $e$ Tecnologia de Alimentos, v. 27, n. 3, p. 451-455, doi:10.1590/S0101-20612007000300005, 2007.

COUTINHO, M. R.; CONCEIÇÃO, W. A. S.; PARAÍSO, P. R.; ANDRADE, C. M. G.; OMOTO, E. S.; JORGE, R. M. M.; MACIEL FILHO, R.; JORGE, L. M. M. Application of the Hsu model to soybean grain hydration. Food Science and Technology (Campinas), v. 30, n. 1, p. 19-29, doi:10.1590/S0101-20612010005000019, 2010.

DORMAND, J. R. e PRINCE, P. J. A family of embedded Runge-Kutta formulae. Journal of Computational and Applied Mathematics, v. 6, n. 1, p. 19-26, doi:10.1016/0771050X(80)90013-3, 1980.

GURIQBAL, S. ED. The soybean: botany, production and uses. Wallingford: CABI, 2010.

LEVENBERG, K. A method for the solution of certain problems in least squares. Quart. Applied Math., v. 2, p. 164-168, 1944.

LUTZ, I. A. Normas analíticas do Instituto Adolfo Lutz. São Paulo: [s.n.], 1985.

MARQUARDT, D. W. An Algorithm for Least-Squares Estimation of Nonlinear Parameters. Journal of the Society for Industrial and Applied Mathematics, v. 11, n. 2, p. 431-441, doi:10.1137/0111030, 1963.

NICOLIN, D. J. Hidratação de grãos: Influência das condições de contorno e da variacão de volume sobre modelos matemáticos.UEM - Universidade Estadual de Maringá - [S.1.]. 2012.

NICOLIN, D. J.; COUTINHO, M. R.; ANDRADE, C. M. G.; JORGE, L. M. M. Soybean Hydration: Investigation of Distributed Parameter Models With Respect To Surface Boundary Conditions. Chemical Engineering Communications, v. 200, n. 7, p. 959-976, doi:10.1080/00986445.2012.732831, 2013.

OMOTO, E. S.; ANDRADE, C. M. G.; JORGE, R. M. M.; COUTINHO, M. R.; PARAÍSO, P. R.; JORGE, L. M. M. Modelagem matemática e análise da hidratação de grãos de ervilha. Ciência e Tecnologia de Alimentos, v. 29, n. 1, p. 12-18, doi:10.1590/S0101-20612009000100003, 2009.

PAN, Z. e TANGRATANAVALEE, W. Characteristics of soybeans as affected by soaking conditions. LWT - Food Science and Technology, v. 36, n. 1, p. 143-151, doi:10.1016/S00236438(02)00202-5, 2003.

PELEG, M. An Empirical Model for the Description of Moisture Sorption Curves. Journal of Food Science, v. 53, n. 4, p. 1216-1217, doi:10.1111/j.1365-2621.1988.tb13565.x, 1988.

PILOSOF, A. M. R.; BOQUET, R. e BARTHOLOMAI, G. B. Kinetics of Water Uptake by Food Powders. Journal of Food Science, v. 50, n. 1, p. 278-279, doi:10.1111/j.13652621.1985.tb13334.x, 1985.

PINTO, J. C. e LAGE, P. L. da C. Métodos numéricos em problemas de engenharia química. Primeira ed. Rio de Janeiro: E-papers Serviços Editoriais Ltda, 2001. p. 316

SINGH, B. P. N. e KULSHRESTHA, S. P. Kinetics of Water Sorption by Soybean and Pigeonpea Grains. Journal of Food Science, v. 52, n. 6, p. 1538-1541, doi:10.1111/j.13652621.1987.tb05874.x, 1987. 